\title{
Microwave Impedance Spectroscopy and Temperature Effects on the Electrical Properties of Au/BN/C Interfaces
}

\author{
Hazem K. Khanfar, ${ }^{1}$ A. F. Qasrawi, ${ }^{2}$ and Yasmeen Kh. Ghannam ${ }^{2}$ \\ ${ }^{1}$ Department of Telecommunication Engineering, Arab-American University, Jenin, State of Palestine \\ ${ }^{2}$ Department of Physics, Arab-American University, Jenin, State of Palestine \\ Correspondence should be addressed to Hazem K. Khanfar; hazem.khanfar@aauj.edu
}

Received 11 November 2016; Revised 5 January 2017; Accepted 24 January 2017; Published 26 February 2017

Academic Editor: Gerard Ghibaudo

Copyright (c) 2017 Hazem K. Khanfar et al. This is an open access article distributed under the Creative Commons Attribution License, which permits unrestricted use, distribution, and reproduction in any medium, provided the original work is properly cited.

In the current study, an Au/BN/C microwave back-to-back Schottky device is designed and characterized. The device morphology and roughness were evaluated by means of scanning electron and atomic force microscopy. As verified by the Richardson-Schottky current conduction transport mechanism which is well fitted to the experimental data, the temperature dependence of the currentvoltage characteristics of the devices is dominated by the electric field assisted thermionic emission of charge carriers over a barrier height of $\sim 0.87 \mathrm{eV}$ and depletion region width of $\sim 1.1 \mu \mathrm{m}$. Both the depletion width and barrier height followed an increasing trend with increasing temperature. On the other hand, the alternating current conductivity analysis which was carried out in the frequency range of 100-1400 MHz revealed the domination of the phonon assisted quantum mechanical tunneling (hopping) of charge carriers through correlated barriers (CBH). In addition, the impedance and power spectral studies carried out in the gigahertz-frequency domain revealed a resonance-antiresonance feature at frequency of $\sim 1.6 \mathrm{GHz}$. The microwave power spectra of this device revealed an ideal band stop filter of notch frequency of $\sim 1.6 \mathrm{GHz}$. The ac signal analysis of this device displays promising characteristics for using this device as wave traps.

\section{Introduction}

Metal-semiconductor-metal (MSM) devices have been in the focus of scientists for decades due to their wide range of applications. They have been used for plasmonic hot-electron photodetection applications [1] like solar blind ultraviolet photodetectors [2]. They are also used as a bistable resistor for digital imaging applications [3]. In addition, the MSM devices are employed as varactors, which can reach cutoff frequency up to $308 \mathrm{GHz}$ [4]. One more accountable add for the applications of these devices is the use as nanoplasmonic waveguides which can find applications in integrated nanophotonic circuits [5].

For ultraviolet applications, wide band gap materials are preferably used to form MSM devices. As a fastresponse flexible ultraviolet photodetector employing a metal-semiconductor-metal structure InGaZnO photodiode is fabricated [6]. The flexible photodetector shows relatively good photoresponse characteristics before and after bending and retains good folding reproducibility after repeated bending up to 500 cycles. More importantly, it shows a fast speed with response and recovery times of $0.8 \mathrm{~ms}$ and $2.0 \mathrm{~ms}, 33.8 \mathrm{~ms}$, being much faster than that of the reported flexible ultraviolet detectors [6]. Boron nitride is also used in the fabrication of MSM devices. Gold-hexagonal boron nitride-gold tunnel junctions are used for conversion of electrons to free-space photons, mediated by resonant slot antennas [7]. They achieved polarized, directional, and resonantly enhanced light emission from inelastic electron tunneling [7]. Also, boron nitride based MSM are highly promising for realizing highly sensitive solid-state thermal neutron detectors with expected advantages resulting from semiconductor technologies, including compact size, light weight, ability to integrate with other functional devices, and low cost [4]. On the other hand, in our previous work [8], an $\mathrm{Ag} / \mathrm{BN} / \mathrm{Ni}$ microwave rejection-band filter was 


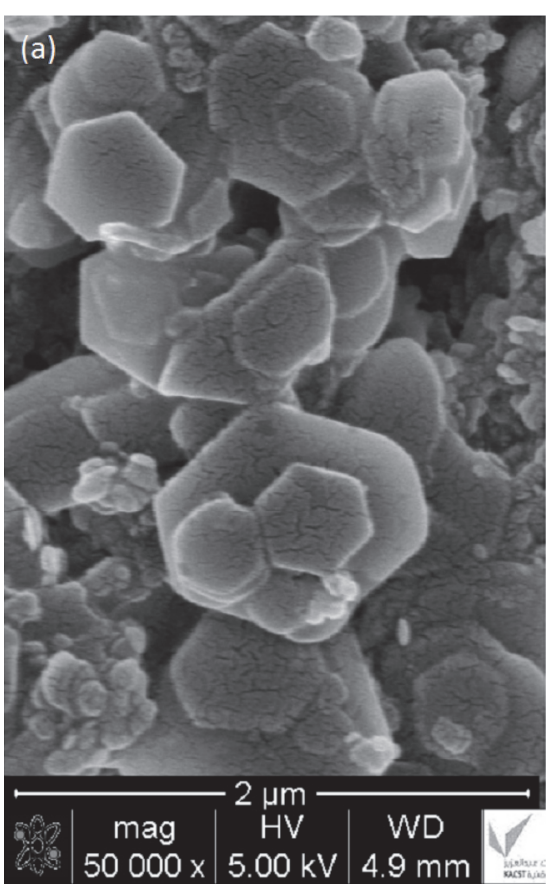

(a)
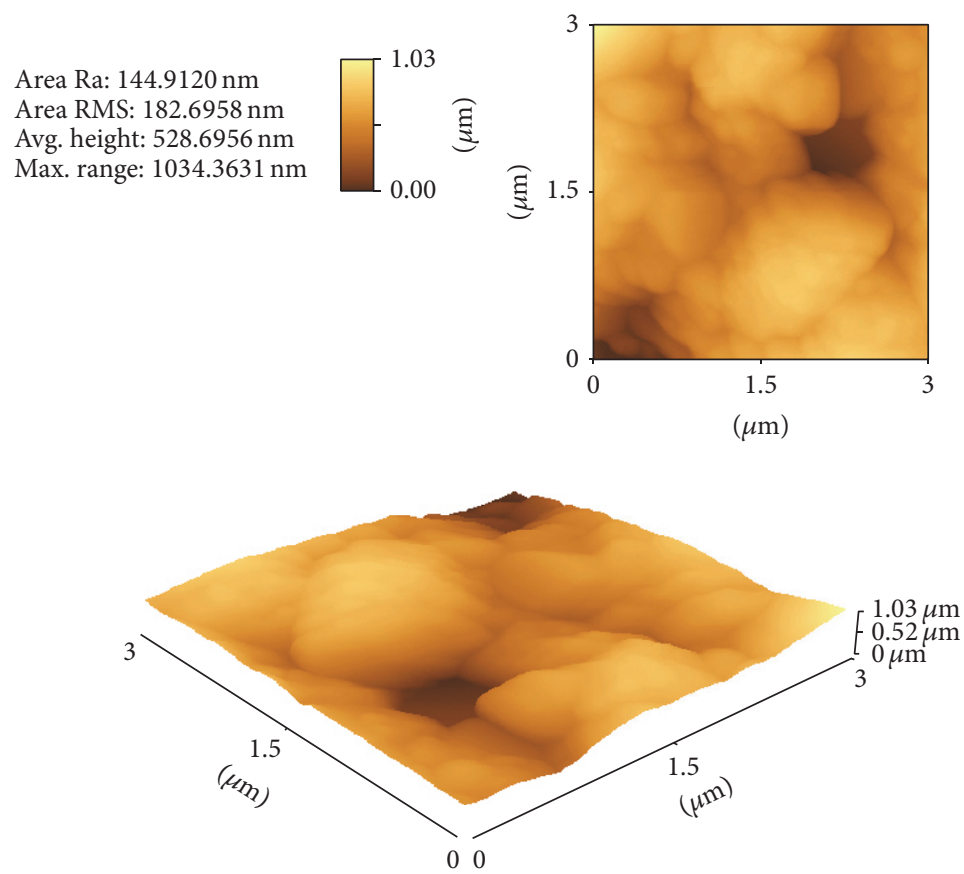

(b)

FIGURE 1: (a) The scanning electron microscopic image for the BN films being magnified 5000 times; (b) the atomic force microscopy images for the BN surface.

designed and characterized. The device was characterized by current-voltage $(I-V)$ characteristics, differential resistance, frequency, and power dependence in the frequency range 1.0$3.0 \mathrm{GHz}[8]$. The above-mentioned technological applications of boron nitride indicate its novelty for microwave sensing. Because the BN exhibits a very high work function of $9.64 \mathrm{eV}$ owing to its wide band gap it easily establishes Schottky barrier with most metals. In addition, recalling that the $\mathrm{Ag} / \mathrm{BN} / \mathrm{Ni}$ device is novel as it was able to screen very weak microwave signals which suits mobile amplifiers at $2.9 \mathrm{GHz}$ [8], here in this work we aim to fabricate new class of these MSM devices for the purpose of optimizing wide range of microwave signals sensing. Particularly, an Au/BN/C MSM band stop filter that works at high temperatures up to $100^{\circ} \mathrm{C}$ is fabricated and characterized. The band stop filter is designed so that it can stop signals at gigahertz frequencies even in high temperature media. The device is characterized by $(I-V)$ characteristics with different temperature in the range of $306-373 \mathrm{~K}$. On the other hand, the device is characterized using an impedance material analyzer and using a $3.0 \mathrm{GHz}$ signal generator and $3.0 \mathrm{GHz}$ spectrum analyzer. The AC current conduction properties of the $\mathrm{Au} / \mathrm{BN} / \mathrm{C}$ device are also explored through the analysis of the frequency dependent electrical conductivity in the frequency range of 100-1800 MHz.

\section{Experimental Details}

The $1.0 \mu \mathrm{m}$ gold substrate was thermally evaporated under a vacuum pressure of $5 \times 10^{-5}$ mbar onto glass slides. The
Alfa Aesar 43773-BN paste is used as a wide band gap semiconductor in the design of $\mathrm{Au} / \mathrm{BN} / \mathrm{C} \mathrm{MSM}$ device. The paste, which was prepared at the Alfa Aesar chemical firm (product number 043773) included a typical solution of $\mathrm{BN}$ nanopowders (50\%) solved in $40 \%$ water and $10 \%$ aluminum phosphate (AlPO4). Further details about the properties of this material were previously given [9]. The BN liquid was poured over the gold substrate, shacked gently, and left to dry for 24 hours. As determined by a digital micrometer, the thickness of the BN layer was $\sim 400 \mu \mathrm{m}$. The scanning electron microscopy images which were recorded with the help of Jeol JSM 7600F microscope and represented in Figure 1(a) display well-shaped hexagonal structured grains of size in the range of $0.60-0.36 \mu \mathrm{m}$. On the other hand, the atomic force microscopy (AFM) images for the same samples which are displayed in Figure 1(b) and evaluated for a surface area of $9.0 \mu \mathrm{m}^{2}$ have shown an average surface roughness $\left(R_{a}\right)$ of $145 \mathrm{~nm}$ and root mean square average of height deviation $\left(R_{\mathrm{MS}}\right)$ of $\sim 183 \mathrm{~nm}$. The deviation in the surface height is small as compared to the sample thickness and represents only about $0.046 \%$ of the total thickness. This result indicates that the metallic layer (carbon) which is located on the top surface of the $\mathrm{BN}$ will represent homogenous and stable electrode area. The $\mathrm{Au} / \mathrm{BN}$ layer was heat treated at $530 \mathrm{~K}$ for 15 minutes to guarantee the absence of the moisture between the grains or through the $\mathrm{BN}$ flakes. The contact area of conducting carbon was $\approx 3.14 \times 10^{-2} \mathrm{~cm}^{2}$. The $I-V$ characteristics data were recorded at different temperatures in the range of 306$373 \mathrm{~K}$ using Keithley 230 programmable voltage source and Keithley 6485 picoammeter. The temperature was varied in 


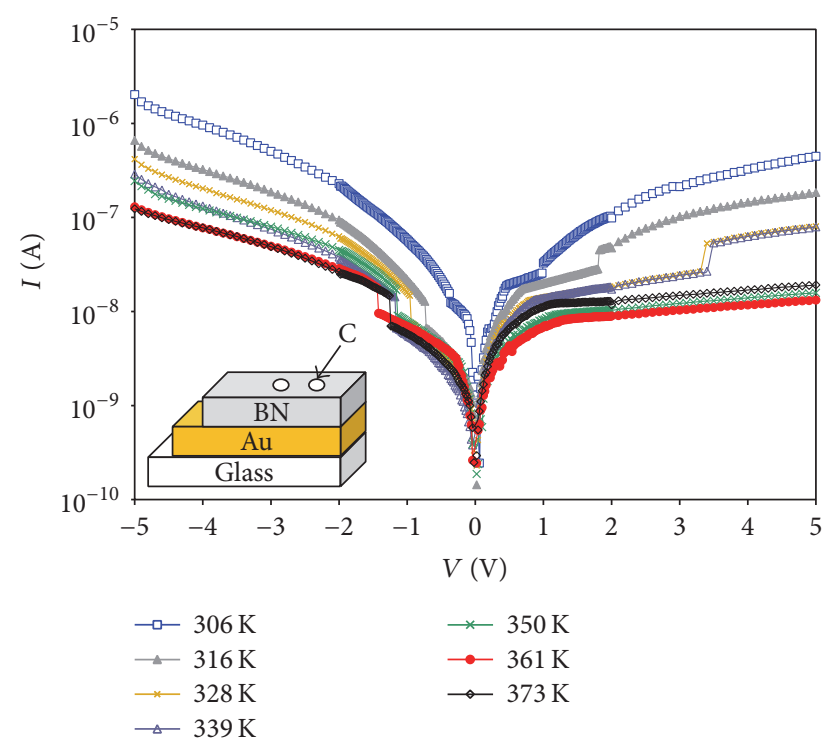

Figure 2: The $I-V$ characteristics and geometrical design of $\mathrm{Au} / \mathrm{BN} / \mathrm{C}$ device.

room atmosphere to allow observation of device natural operational conditions. For this reason, the temperature stability with atmosphere was controlled by the most accurate temperatures for at least 10-minute period of time. After each heating cycle within the mentioned range, the current-voltage characteristic curve was compared to the previous cycle to guarantee operation stability. On the other hand, a 4291B RF Impedance/Material Analyzer was used to register the dielectric data. Also, an N9310A RF Signal Generator (9 kHz$3 \mathrm{GHz}$ ) with Instek GSP $8303 \mathrm{GHz}$ Spectrum Analyzer is used to collect the power spectrum data.

\section{Results and Discussion}

As the metal work functions of the gold and carbon are $5.34 \mathrm{eV}$ and $5.1 \mathrm{eV}$, respectively, and the $\mathrm{BN}$ is of p-type (as determined by the hot probe technique) with barrier height of $9.64 \mathrm{eV}$ [9], the $\mathrm{Au} / \mathrm{BN}$ represent a Schottky diode and the $\mathrm{C} / \mathrm{BN}$ represent another Schottky diode. The presentation of both Schottky-type metals on the front and rare part of the $\mathrm{BN}$ makes the $\mathrm{Au} / \mathrm{BN} / \mathrm{C}$ device structure represent a metalsemiconductor-metal (MSM) back-to-back Schottky device. Figure 2 shows the $I-V$ characteristics of the Au/BN/C MSM device being registered in the temperature range of 300$375 \mathrm{~K}$. As the curves, which are plotted on a logarithmic scale reads $\left(I \propto e^{q V / k T}\right)$, increasing the temperature decreases the current values. As, for example, at a forward biasing voltage of $2.0 \mathrm{~V}$ and temperature of $306 \mathrm{~K}$, the current value is $0.99 \mathrm{nA}$, when the temperature is increased to $339 \mathrm{~K}$, the current becomes $0.17 \mathrm{nA}$ and it reaches a value of $0.12 \mathrm{nA}$ at $T=373 \mathrm{~K}$. In addition, the forward biasing of device exhibits a temperature dependent switching property. The switching voltage $\left(V_{S}\right)$ from low injected current to high injected current increases with increasing temperature. Namely, $V_{S}$ increase from $1.0 \mathrm{~V}, 1.8 \mathrm{~V}, 3.4 \mathrm{~V}$, to $3.5 \mathrm{~V}$ as the temperature increases from $306,316,328$, to $339 \mathrm{~K}$, respectively. At higher temperatures above $339 \mathrm{~K}$, the switching feature was not detectable due to measuring range limitations. In the reverse bias direction $\mathrm{V}_{S}$ exhibited negative values of $0.38,0.68,0.94$, $1.08,1.18,1.42$, and $1.22 \mathrm{~V}$ as the temperature is raised from $306,316,328,339,350,361$, to $373 \mathrm{~K}$, respectively. Following the previous investigation $[8,10]$, the $I-V$ characteristics of the forward bias current were analyzed in accordance with the Richardson-Schottky model that assumes direct current electrical conduction through electric field assisted thermionic emission. The current depends on the square root of the voltage and can be presented by the following equation [10]:

$$
I=A A^{* *} T^{2} \exp \left(-\frac{\phi_{b}}{k T}\right) \exp \left(\frac{-e \beta_{s} \sqrt{V}}{\left(k T \sqrt{w_{o}}\right)}\right) .
$$

The analysis of the $I-V$ with the help of this equation reveals the depletion width $\left(w_{o}\right)$ from the slope and the value of the field independent barrier height $\left(\phi_{b}\right)$ to the electron motion from the intercept of the $\ln (I)-\sqrt{V}$ variation through the respective relations:

$$
\begin{aligned}
w_{o} & =\left(\frac{-e \beta_{s}}{k T(d(\ln (I)) / d(\sqrt{V}))}\right), \\
\phi_{b} & =-\frac{k T}{e}\left[\frac{\ln (I(V=O))}{A A^{* *} T^{2}}\right] .
\end{aligned}
$$

In (2) and (3), $A$ is the device area, $A^{* *}=90$ [11] is Richardson constant, $\beta_{s}=\sqrt{e /\left(4 \pi \varepsilon_{o} \varepsilon_{r}\right)}=1.39 \times 10^{-4}(\mathrm{Vcm})^{1 / 2}$ is Richardson-Schottky coefficient, and $\varepsilon_{r}=7.5$ [11] is the optical dielectric constant of the material. The temperature dependence of the values of the depletion width $\left(w_{o}\right)$ and $\phi_{b}$ are shown in Figures 3(a) and 3(b). As it is readable from Figure 3(a), the junction width changes from $1.14 \mu \mathrm{m}$ to 2.26 and reaches $3.3 \mu \mathrm{m}$ as the temperature increased from 306 to 339 and reaches $361 \mathrm{~K}$, respectively. Consistently, the values of the barrier height which are displayed in Figure 3(b) increased from 0.872 to 0.913 and reached $0.93 \mathrm{eV}$ as the temperature increased from 306 to 339 and reached $361 \mathrm{~K}$, respectively. The rate of change of the depletion width and energy barrier height with temperature in accordance with the linear fitting of the data (shown by solid lines in Figure 3) are $w_{o}=0.037 T-10.20(\mu \mathrm{m})$ and $\varphi_{o}(T)=7.08 \times$ $10^{-4} \mathrm{~T}+0.68(\mathrm{eV})$. The increase in the barrier height and in the depletion width of the $\mathrm{Au} / \mathrm{BN} / \mathrm{C}$ devices with increasing temperature could be ascribed to many physical reasons like the increase in the electron-hole recombination rate, the thermal expansion, and the lattice constants variations with temperature. The hexagonal $\mathrm{BN}$ is reported [12] to exhibit a temperature dependent lattice constants of $c=$ $6.6516 \AA+2.74 \times 10^{-4} T\left(\AA^{\circ} \mathrm{C}^{-1}\right)$ and $a=2.50424 \AA-$ $7.42 \times 10^{-6} T\left(\AA^{\circ} \mathrm{C}^{-1}\right)+4.79 \times 10^{-9} \mathrm{~T}^{2}\left(\AA^{\circ} \mathrm{C}^{-2}\right)$. The lattice parameters along the $c$-axis and along the $a$-axis of the BN polycrystals increased from 6.7338 to $6.7505 \AA$ and from 2.5024 to $2.5384 \AA$ as the temperature increases from 300 to $360 \mathrm{~K}$ for each unit cell. On the other hand, the excitonic energy band gap of the hexagonal $\mathrm{BN}$ is reported 


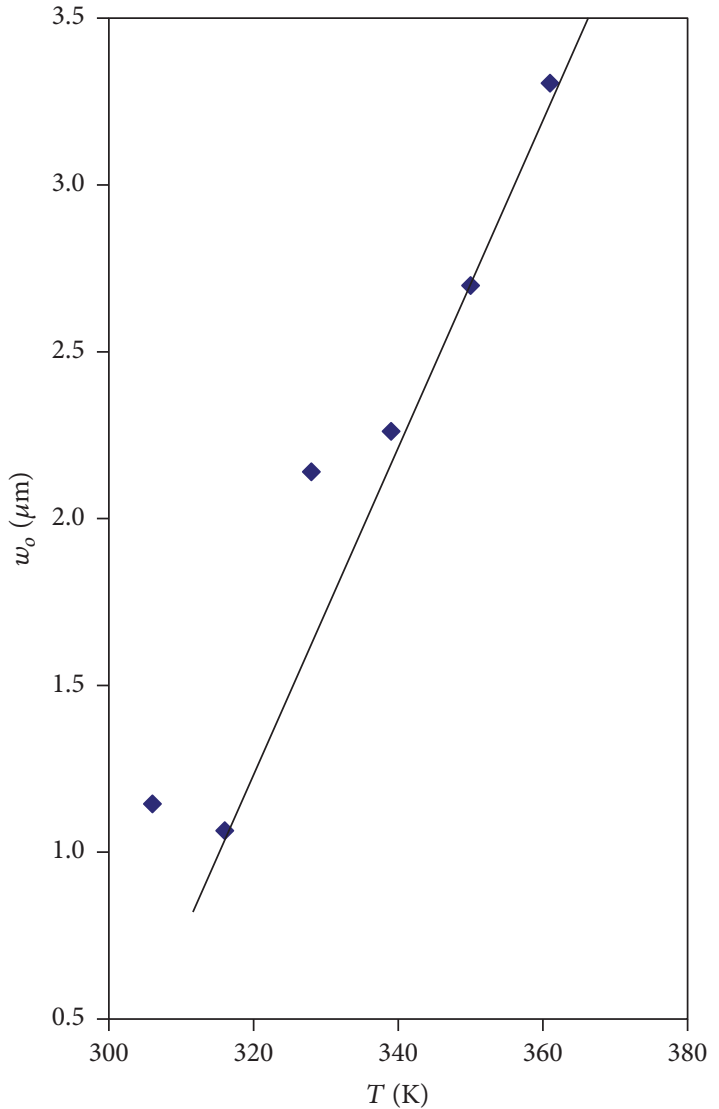

(a)

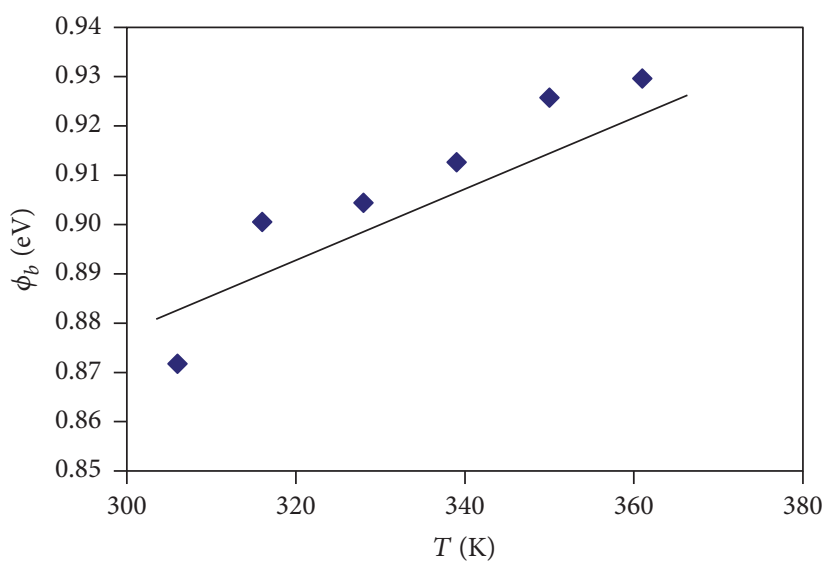

(b)

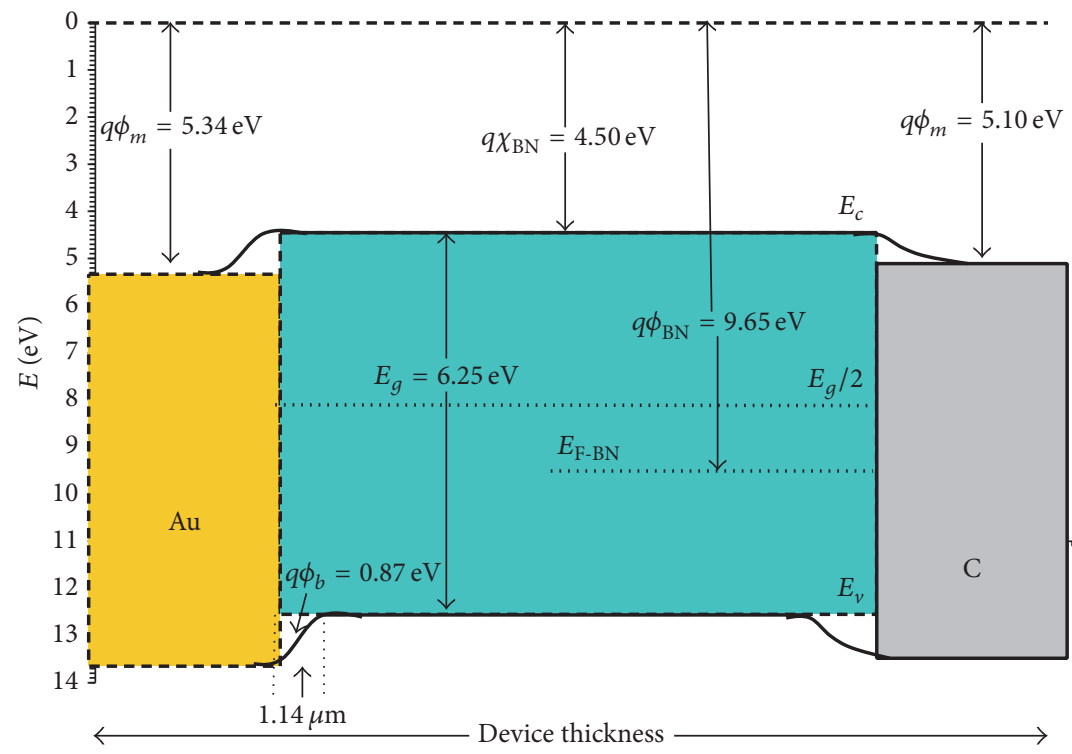

(c)

Figure 3: The temperature dependence of (a) the depletion region width and (b) the barrier height of the Au/BN/C device. (c) The energy band diagram of the device. In the diagram $\phi_{m}, q \chi, q \phi_{b}, E_{c}, E_{v}, E_{\mathrm{F}-\mathrm{BN}}$, and $E_{g}$ are the metal work function, electron affinity, barrier height, conduction band energy level, valence band energy level, the Fermi level of $\mathrm{BN}$, and the energy band gap, respectively. 
to exhibit a negative temperature coefficient of $\sim 4.3 \times 10^{-4}$ $\mathrm{eV} /{ }^{\circ} \mathrm{K}[13]$. The latter value indicates an expected effect on the device parameters $\left(\varphi_{b}, w_{o}\right)$ associated with the narrowing of the energy band gap of $\mathrm{BN}$. Although the shrinkage in the energy band gap may be of less effect, it indicates an increase in the number of charge carriers that are transported from the valence to the conduction band which means more electron-hole recombination ratios and wider depletion region. Similar characteristics which involve increasing the barrier height with increasing temperature were observed for $\mathrm{Au} / \mathrm{n}-\mathrm{GaAs} / \mathrm{n}-\mathrm{Ge}$ diodes [14]. The increase in the barrier height with increasing temperature was ascribed to the electron-hole recombination dynamics and to the electric field dependence of the barrier height (similar to our case). In another study, the increase in the barrier height with increasing temperature was also assigned to the existence of double Gaussian distribution of energy barriers represented by the screened mean barrier height in the sample. The double Gaussian distribution of energy barriers results from the barrier height inhomogeneity at the metal/semiconductor (MS) interface.

The energy band diagram of the device is shown in Figure 3(c). The working principle of the $\mathrm{Au} / \mathrm{BN} / \mathrm{C}$ device which represents two Schottky barriers connected back to back is that the biasing of any polarity will put one Schottky barrier in the reverse direction ( $\mathrm{Au}$ side as a cathode) and the other in the forward direction (C-side as an anode) [10]. While the depletion width of the forward biased diode narrows, the depletion width of the reverse biased diode widens. Thus, the measured width which is presented in Figure 3(a) indicated the effective depletion region width of the device [10]. At particular temperature, $306 \mathrm{~K}$, for example, the appearance of the large reverse current which lowers the rectification ratio and makes the $I-V$ characteristic appear as if it was ohmic is explained by means of the current transport mechanism through the two diodes which have depletion widths $W_{D 1}$ and $W_{D 2}$. When one of the diodes is forward biased, its depletion width $\left(w_{D 1}\right)$ narrows, causing large forward and low reverse current for low applied voltages. The narrow barrier makes the charge transport via tunneling process preferable [10]. In this case the current conduction mechanism is governed by electric field assisted tunneling of charged particles through the narrow barrier consistent with (1). On the other side of the device, the second diode is reverse biased and its depletion width $\left(w_{D 2}\right)$ widens with increasing voltage. This behavior also lowers the values of the reverse current compared to the forward one. However, as the voltage is further increased, the depletion region of second diode reaches the first region leading to the flat band condition, in which the ohmic nature of contact dominates [10]. Another point that is worth of consideration is that when the reach-through voltage is reached, the exponentially increasing current with increasing applied voltage is also affected by electron-hole generation-recombination, avalanche breakdowns, and image force lowering potentials processes [10] leading to the observed high reverse current.

It is also worth noting that the current-voltage characteristics of the device exhibit low rectification ratio associated with high resistance values $\left(R_{s}>10 \mathrm{M} \Omega\right)$. The rectification ratio varies in the range of 5-11. This behavior which was also observed for some organic/inorganic heterojunctions was assigned to the recombination losses caused by surface trap states [15]. The leakage current is observed to be affected by the distribution of defects and the quality of the interfaces. In addition, the lattice mismatch between the hexagonal and the face centered cubic gold film and diamond structured carbon film could be a main reason as the force confinement of electrons and holes in different spaces.

Figure 4 shows the ac signal analysis of $\mathrm{Au} / \mathrm{BN} / \mathrm{C}$ MSM device being recorded with the help of a $1.8 \mathrm{GHz}$ impedance/material analyzer under no biasing conditions. The parallel capacitance spectra of the device which are illustrated in Figure 4(a) slightly decreased with increasing frequency up to $1594 \mathrm{MHz}$. For larger frequencies there appear resonance (series) antiresonance (parallel) oscillatory modes at two different frequency regions. The series resonance $\left(f_{s}\right)$ appears at 1620 and $1647 \mathrm{MHz}$, while the parallel resonance $\left(f_{p}\right)$ is apparent at 1611 and at $1629 \mathrm{MHz}$. The resonanceantiresonance modes of oscillation in the $\mathrm{Au} / \mathrm{BN} / \mathrm{C}$ device are of importance as they can be used as wave traps, which are sometimes inserted in series with antennas of radio receivers to block the flow of alternating current at the frequency of an interfering station, while allowing other frequencies to pass [16].

The resonance-antiresonance modes of oscillation in the $\mathrm{Au} / \mathrm{BN} / \mathrm{C}$ device can be modeled as two parallel arms: the motional arm and the static arm. The motional arm is represented by a series RLC circuit while the static arm is represented by a shunt capacitor. The reactive impedance $\left(X_{m}\right)$ of the motional arm can be either positive or negative depending on the frequency; also at specific frequency series resonance $\left(f_{s}\right)$ the reactive impedance is minimum $\left(X_{m} \approx 0\right)$. The antiresonance $f_{p}$ (resonance of high impedance) occurs at a particular frequency when the impedance of the motional arm is very high compared to the impedance of the static arm and the device can be modeled as shunt capacitor. At this frequency $\left(f_{p}\right)$ the total admittance $(Y)$ of the device is minimum $\left(Y=Y_{\text {motional }}+Y_{\text {static }} \approx 0\right)$ which indicates that the reactive impedance of the static arm and the reactive impedance of the motional arm are equivalent in magnitude and opposite in signs. On the other hand, Figure 4(b) shows the parallel resistance of the device versus the frequency. The resistance decreases from $6365.42 \Omega$ at $100 \mathrm{MHz}$ to 448.14 at $1800 \mathrm{MHz}$. The trend of the impedance looks like the capacitive part of the device and can be ascribed to the same dynamics. The total effect which is known as the impedance $(-Z-)$ spectra of the resistive and reactive parts (inductive and capacitive) is plotted in Figure 4(c). It is noticed that as the frequency increases, $|Z|$ decreases. Namely, $|Z|$ decreases from $548.59 \Omega$ at $100 \mathrm{MHz}$ to $33.94 \Omega$ at $1800 \mathrm{MHz}$. This indicates that the capacitive part is more dominating than the inductive part of the impedance load.

To give more deep significance to the impedance spectral data, the alternating current conductivity $\left(\sigma_{\mathrm{ac}}\right)$ is calculated from the conductance spectra $\left(1 / R_{p}\right)$. The conductivity spectrum which is displayed in Figure $4(\mathrm{~d})$ exhibits an increasing trend of variation with increasing frequency. In the frequency range of $100-1400 \mathrm{MHz}$, the $\sigma_{\mathrm{ac}}-f$ variation 


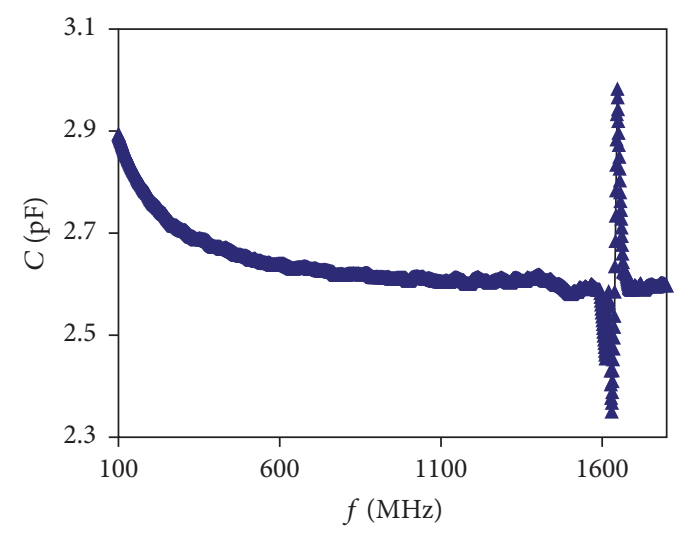

(a)

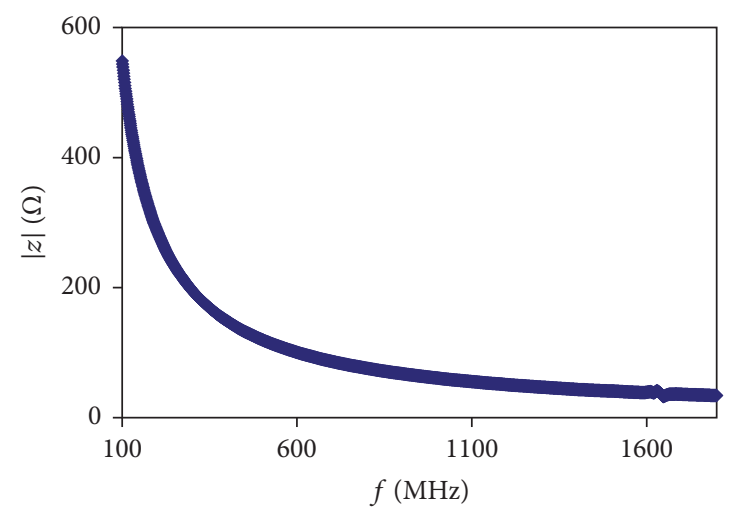

(c)

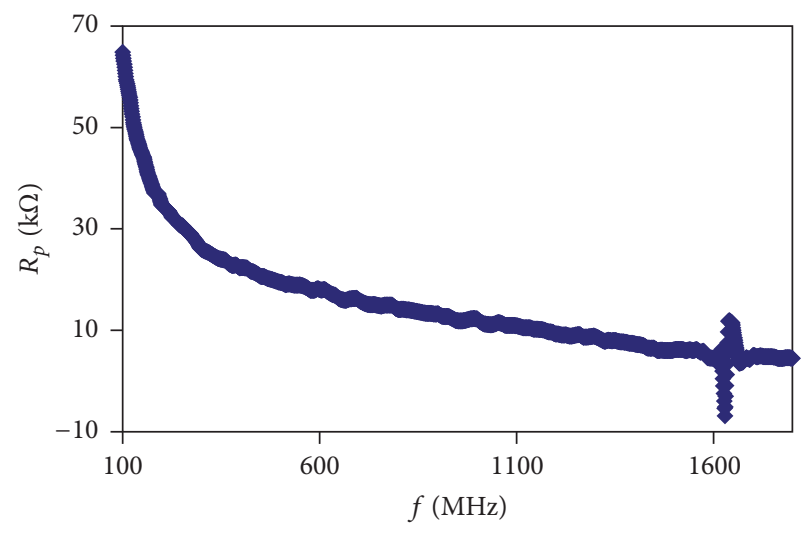

(b)

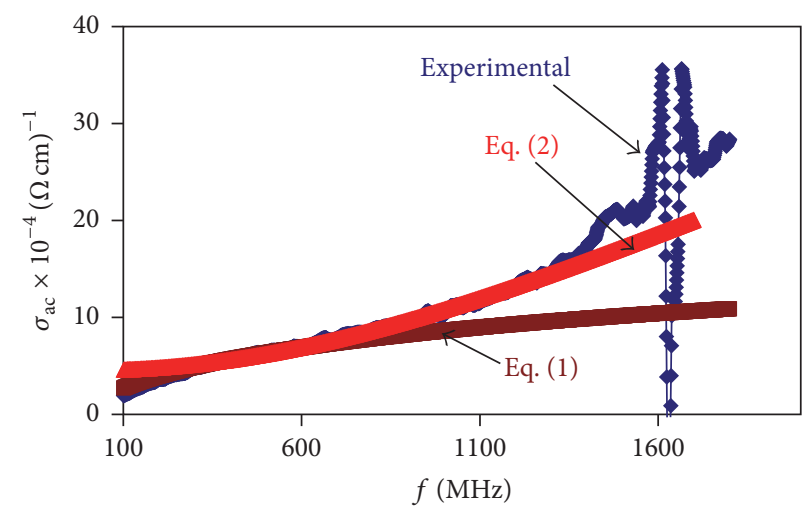

(d)

FIgURE 4: (a) The capacitance, (b) the parallel resistance, (c) the total impedance, and (d) the conductivity spectra for the Au/BN/C device.

could be represented by the relation $\sigma_{\mathrm{ac}} \propto w^{s}$. The angular frequency $(w)$ exponent $(s)$ for the $\mathrm{Au} / \mathrm{BN} / \mathrm{C}$ device is 0.79 . The increase of $\sigma_{\mathrm{ac}}$ with frequency in the frequency range of $100-1400 \mathrm{MHz}$ is an indication of the domination of the correlated barrier hopping mechanism $(\mathrm{CBH})$ in the samples $[17,18]$. In accordance with this model the $s$ value takes the form $s=1-6 k T /\left(W_{M}+k T \ln \left(w \tau_{o}\right)\right)$ with $W_{M}$ and $\tau_{o}$ being the maximum barrier height at infinite intersite separation (binding energy of carrier in its localized site) and the relaxation time, respectively. The $\mathrm{CBH}$ model assumes that the electrons hop between pairs of localized states at the Fermi level and relates the conductivity to the density of states $\left(N\left(E_{F}\right)\right)$ at the Fermi level through the relation $[17,18]$

$$
\sigma_{\mathrm{ac}}=\left(\frac{\pi}{3}\right) e^{2} k T w \xi^{5}\left(N\left(E_{F}\right)\right)^{2}\left(\ln \left(\frac{v_{\mathrm{ph}}}{w}\right)\right)^{4} .
$$

In this relation, $e$ is the electronic charge, $\xi=10 \AA$ is the typical localization length, and $v_{\mathrm{ph}}=10^{12} \mathrm{~Hz}$ is the phonon frequency. The fitting of the experimental data of conductivity in accordance with (1) that reveals the exact frequency exponent parameter $(s=0.79)$ which is shown in Figure 4(d) as brown colored allowed determining the value of $N\left(E_{F}\right)$ as $5.40 \times 10^{18} \mathrm{eV}^{-1} \mathrm{~cm}^{-3}$ in the frequency range of $100-850 \mathrm{MHz}$. The corresponding maximum barrier heights at infinite intersite separation $W_{M}$ and $\tau_{o}$ are found to be
$0.97 \mathrm{eV}$ and $50 \mathrm{ps}$, respectively. The values of $W_{M}$ and $\tau_{o}$ are in good consistency with the literature data $[17,18]$.

Recalling that the AC conductivity increases with increasing frequency as long as the frequency of the field is lower than the charge carrier jump frequency in solids, one may reproduce the experimental data in a wider range of frequency $(100-1400 \mathrm{MHz})$ assuming the existence of the high $\sigma_{\mathrm{ac}}(H)$ and low $\sigma_{\mathrm{ac}}(L)$ limits to the $\mathrm{CBH}$ conductivity in accordance with the relation

$$
\sigma_{\mathrm{ac}}=\sigma_{\mathrm{ac}}(H)+\frac{\sigma_{\mathrm{ac}}(L)-\sigma_{\mathrm{ac}}(H)}{1+\left(w \tau_{\mathrm{o}}\right)^{2}} .
$$

The fitting curve which is shown as red colored in Figure 4(d) can be reproduced by the substitution of $\sigma_{\mathrm{ac}}(L)=$ $4.50 \times 10^{-4}(\Omega \mathrm{cm})^{-1} \quad \sigma_{\mathrm{ac}}(H)=7.00 \times 10^{-3}(\Omega \mathrm{cm})^{-1}$. The excellent consistency between the experimental and theoretical data ensures the correctness of the domination of the $\mathrm{CBH}$ conduction mechanism in the $\mathrm{Au} / \mathrm{BN} / \mathrm{C}$ devices.

It is worth notifying that the impedance spectra which were discussed in this work related to no electric field biasing. The correlated barrier hopping conduction mechanism is a phonon assisted quantum mechanical tunneling process that takes place between defect states and/or in the depletion regions of the device at the $\mathrm{Au} / \mathrm{BN}$ and $\mathrm{C} / \mathrm{BN}$ interfaces. The electric field assisted tunneling conduction mechanism which was determined from the direct current analysis 


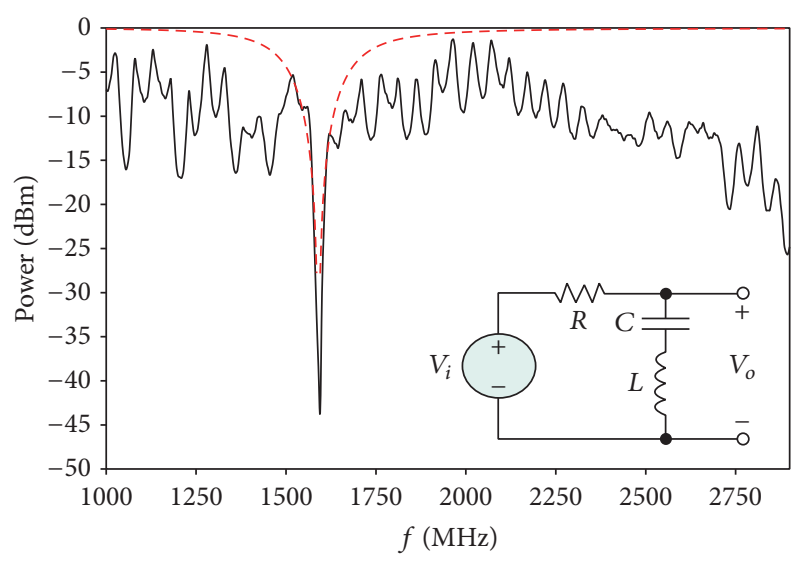

FIGURE 5: Gain-frequency dependence of the Au/BN/C device. Dashed plot is the transfer function of the modeled RLC circuit.

revealed a room temperature barrier height of $0.87 \mathrm{eV}$. This value is less than the maximum barrier height at infinite intersite separation which is determined as $0.97 \mathrm{eV}$ indicating the correctness of the evaluation procedure of the conduction mechanism. It is also important to notice that, for applied frequencies larger than $1400 \mathrm{MHz}$, the dynamics of the conductance changes abnormally as a result of the resonance-antiresonance phenomenon which is observed near $1600 \mathrm{MHz}$. This series-parallel resonance case at this frequency is also observed in many wide gap glassy materials including the glass itself (measured in our laboratory). However, because the glass is coated with metal and metal is directly connected to the fixture of the impedance analyzer, the resonance case is mostly due to the $\mathrm{BN}$ itself. Considering the peak which appears at $1600 \mathrm{MHz}$ in the negative parallel resistance mode to be due to the $\mathrm{BN}$, the negative resistance property and the peak itself could have originated from the gate and applied biases as was also observed for the boron nitride-graphene heterostructure [19]. The gate behaves like Fabry-Perot interference which is suppressed by the electronphonon scattering and the applied biases that represent an in-plane wave vector matching when the Dirac points of electrodes align. It is reported that the hexagonal BN layers can induce an asymmetry in the $I-V$ characteristics which can be modulated by the applied bias [19]. In another work which concerns the negative resistance and negative capacitance in $\mathrm{Au} / \mathrm{ZnO} / \mathrm{n}-\mathrm{GaAs}$ Schottky barrier diodes, the increment of negative capacitance (values by high frequency at forward biases) was assigned to the series resistance, interface states, and interfacial layer.

The appearance of the biasing voltage dependent resonance peak in the dielectric spectra which was also observed in the frequency range of $1 \mathrm{~K}-1 \mathrm{MHz}$ for the graphene oxidedoped praseodymium barium cobalt oxide nanoceramics was attributed to the particular distribution of interface states located between Au and interfacial layer and to the interfacial polarization [20].

As a complementary work, Figure 5 shows the output gain versus input frequency being recorded with the help of a $3.0 \mathrm{GHz}$ power spectrum and $3.0 \mathrm{GHz}$ signal generator; the output gain demonstrates the band reject filter with central frequency of $1595 \mathrm{MHz}$. The value of the critical frequency (known as notch frequency) is consistent with that detected from the capacitance spectra at the resonanceantiresonance frequency range. This consistency between the two measuring techniques indicates that the $\mathrm{Au} / \mathrm{BN} / \mathrm{C}$ device is ideal for use as wave traps in the microwave frequency range. The two terminal simple devices which exhibit the behavior of a band reject filter can be modeled using RLC circuit as shown in the inset of Figure 5. The transfer function of the modeled RLC filter circuit is given by [21]

$$
G=\frac{\eta^{2}+\left(w^{*}\right)^{2}}{\eta^{2}+2 \gamma w^{*} \eta+\left(w^{*}\right)^{2}},
$$

where $w^{*}=2 \pi f^{*}$ is the angular frequency, $f^{*}=1 /(2 \pi \sqrt{L C})$ is the center frequency, $\eta=2 \pi i f$, and $\gamma=(R / 2) \sqrt{C / L}$ is the damping factor. The modeling of the device which was carried out using the MATLAB program is presented by the dashed line in Figure 5. The line represents the output of the RLC circuit for fitting parameters of $R=20.0 \Omega, C=1.0 \mathrm{pF}$, and $L=9.96 \mathrm{nH}$. The center frequency $\left(f^{*}\right)$ of this circuit is $1595 \mathrm{MHz}$ with a damping factor of $100.22 \times 10^{-3}$. The fitting parameters values of this device appear to be ideal for use in microwave technology. Such types of filters find applications in telecommunication networks.

It is important to mention that in all parametric calculations the fitting procedures were carried out by a special high-convergence minimization program that makes use of regression and residual sums of squares $\left(R^{2}\right)$, coefficient of determination, and residual mean squares statistical analysis. The errors in the data were evaluated to be $4-12 \%$. Typical best fits for the experimental data and its related modeling are illustrated in Figure 5. All the other calculated slopes (shown in Figures 3(a) and 3(b)) were restricted to give a residual sum of squares $R^{2}=0.97$.

\section{Conclusions}

In this work a new class of $\mathrm{BN}$ based devices is produced and characterized. The device was found to be properly operated at temperatures up to $100^{\circ} \mathrm{C}$. The temperature effect of this device is explored by means of the currentvoltage characteristics which revealed an increasing barrier height and depletion width with increasing temperature. The impedance spectroscopy which was analyzed in the frequency range of $100-1800 \mathrm{MHz}$ allowed determining the current conduction mechanism by correlated barrier hopping. The AC signal analysis of the $\mathrm{Au} / \mathrm{BN} / \mathrm{C}$ device displayed resonance-antiresonance physical phenomena at frequency of $\sim 1.6 \mathrm{GHz}$. The device wave trapping property was verified by the input of an ac signal in the suitable frequency domain and found to behave as an ideal narrow band stop filter with promising features for use in microwave communication technology. 


\section{Competing Interests}

The authors declare that there is no conflict of interests regarding the publication of this paper.

\section{References}

[1] K. Wu, Y. Zhan, C. Zhang, S. Wu, and X. Li, "Strong and highly asymmetrical optical absorption in conformal metal-semiconductor-metal grating system for plasmonic hotelectron photodetection application," Scientific Reports, vol. 5, Article ID 14304, 2015.

[2] W. Zhang, J. Xu, W. Ye et al., "High-performance AlGaN metalsemiconductor-metal solar-blind ultraviolet photodetectors by localized surface plasmon enhancement," Applied Physics Letters, vol. 106, no. 2, 2015.

[3] M. J. Kumar, M. Maram, and P. P. Varma, "Schottky biristor: a metal-semiconductor-metal bistable resistor," IEEE Transactions on Electron Devices, vol. 62, no. 7, pp. 2360-2363, 2015.

[4] D. M. Geum, S. H. Shin, S. M. Hong et al., "Metalsemiconductor-metal varactors based on InAlN/GaN heterostructure with cutoff frequency of $308 \mathrm{GHz}$," IEEE Electron Device Letters, vol. 36, no. 4, pp. 306-308, 2015.

[5] Y. Fang and M. Sun, "Nanoplasmonic waveguides: towards applications in integrated nanophotonic circuits," Light: Science and Applications, vol. 4, article e294, 2015.

[6] H. T. Zhou, L. Li, H. Y. Chen, Z. Guo, S. J. Jiao, and W. J. Sun, "Realization of a fast-response flexible ultraviolet photodetector employing a metal-semiconductor-metal structure InGaZnO photodiode," RSC Advances, vol. 5, no. 107, pp. 87993-87997, 2015.

[7] M. Parzefall, P. Bharadwaj, A. Jain, T. Taniguchi, K. Watanabe, and L. Novotny, "Antenna-coupled photon emission from hexagonal boron nitride tunnel junctions," Nature Nanotechnology, vol. 10, no. 12, pp. 1058-1063, 2015.

[8] H. K. Khanfar, "Fabrication and characterization of Ag/BN/Ni microwave rejection-band filters," IEEE Transactions on Electron Devices, vol. 61, no. 6, pp. 2154-2157, 2014.

[9] S. E. Al Garni and A. F. Qasrawi, "Design and characterization of $(\mathrm{Al}, \mathrm{C}) / \mathrm{p}-\mathrm{Ge} / \mathrm{p}-\mathrm{BN} / \mathrm{C}$ isotype resonant electronic devices," Physica Status Solidi (A) Applications and Materials Science, vol. 212, no. 8, pp. 1845-1850, 2015.

[10] S. Sze and K. K. Ng, Physics of Semiconductor Devices, John Wiley \& Sons, Inc., Hoboken, NJ, USA, 2006.

[11] E. W. S. Caetano, V. N. Freire, G. A. Farias, and E. F. Da Silva Jr., "Optical properties of zincblende GaN/BN cylindrical nanowires," Applied Surface Science, vol. 234, no. 1-4, pp. 50-53, 2004.

[12] O. Madelung, Semiconductors: Data Handbook, Springer, Berlin, Germany, 2004.

[13] X. Z. Du, C. D. Fyre, J. H. Edgar, J. Y. Lin, and H. X. Jiang, "Temperature dependence of the energy bandgap of two-dimensional hexagonal boron nitride probed by excitonic photoluminescence," Journal of Applied Physics, vol. 115, no. 5, Article ID 053503, 2014.

[14] M. K. Hudait, P. Venkateswarlu, and S. B. Krupanidhi, "Electrical transport characteristics of Au/n-GaAs Schottky diodes on n-Ge at low temperatures," Solid-State Electronics, vol. 45, no. 1, pp. 133-141, 2001.
[15] M. Soylu, M. Gülen, and S. Sönmezoğlu, "Temperature-dependent model for hole transport mechanism in a poly(1.8diaminocarbazole)/Si structure," Philosophical Magazine, vol. 96, no. 25, pp. 2600-2614, 2016.

[16] D. M. Pozar, Microwave Engineering, Wiley Interscience, 3rd edition, 2005.

[17] A. A. A. Darwish, M. M. El-Nahass, and A. E. Bekheet, "AC electrical conductivity and dielectric studies on evaporated nanostructured InSe thin films," Journal of Alloys and Compounds, vol. 586, pp. 142-147, 2014.

[18] R. Murti, S. K. Tripathi, N. Goyal, and S. Prakash, "Random free energy barrier hopping model for ac conduction in chalcogenide glasses," AIP Advances, vol. 6, no. 3, Article ID 035010, 2016.

[19] Y. Zhao, Z. Wan, X. Xu, S. R. Patil, U. Hetmaniuk, and M. P. Anantram, "Negative differential resistance in boron nitride graphene heterostructures: physical mechanisms and size scaling analysis," Scientific Reports, vol. 5, Article ID 10712, 2015.

[20] A. Kaya, S. Alialy, S. Demirezen, M. Balbaşı, S. Yerişkin, and A. Aytimur, "The investigation of dielectric properties and ac conductivity of $\mathrm{Au} / \mathrm{GO}$-doped $\mathrm{PrBaCoO}$ nanoceramic/n-Si capacitors using impedance spectroscopy method," Ceramics International, vol. 42, no. 2, pp. 3322-3329, 2016.

[21] H. G. Dimopoulos, Analog Electronic Filters: Theory, Design and Synthesis, Springer, Dordrecht, Netherlands, 2012. 


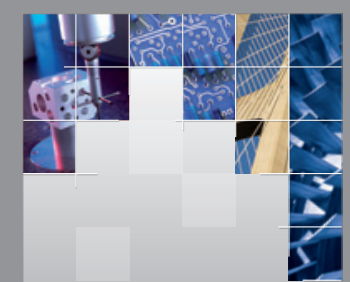

\section{Enfincering}
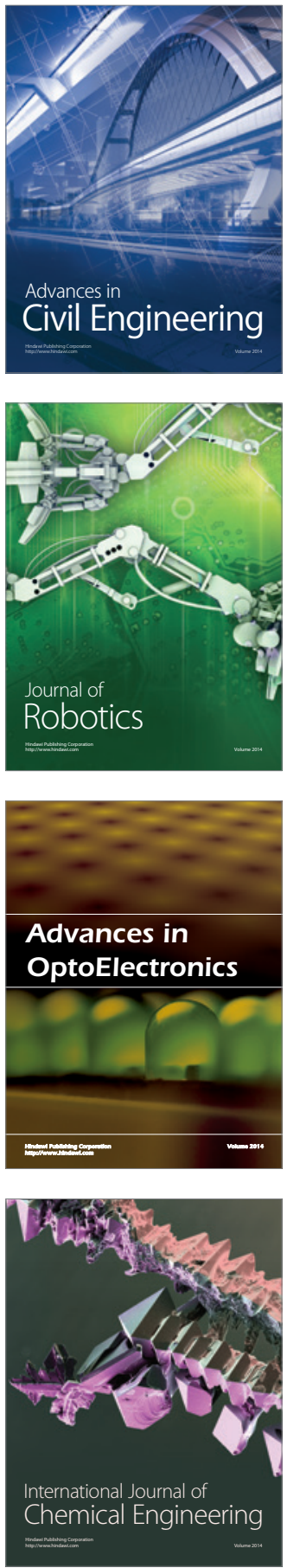

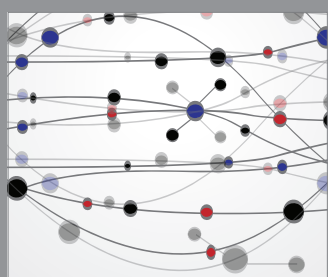

The Scientific World Journal

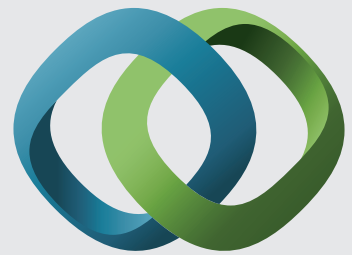

\section{Hindawi}

Submit your manuscripts at

https://www.hindawi.com
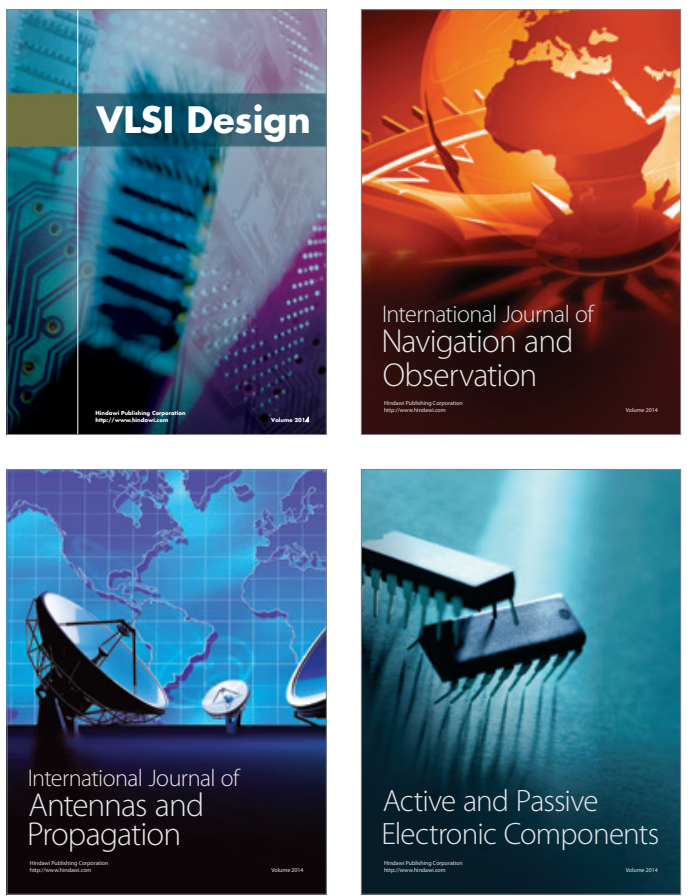
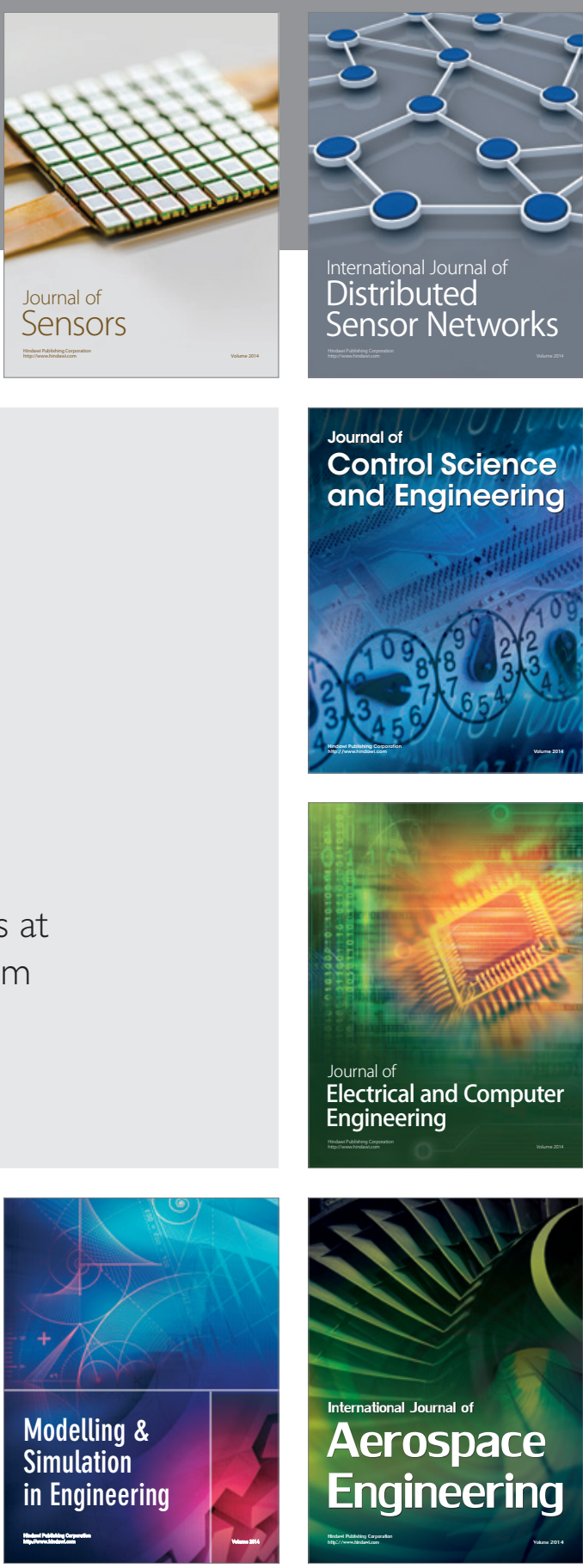

International Journal of

Distributed

Sensor Networks

$-$

Joumal of

Control Science

and Engineering
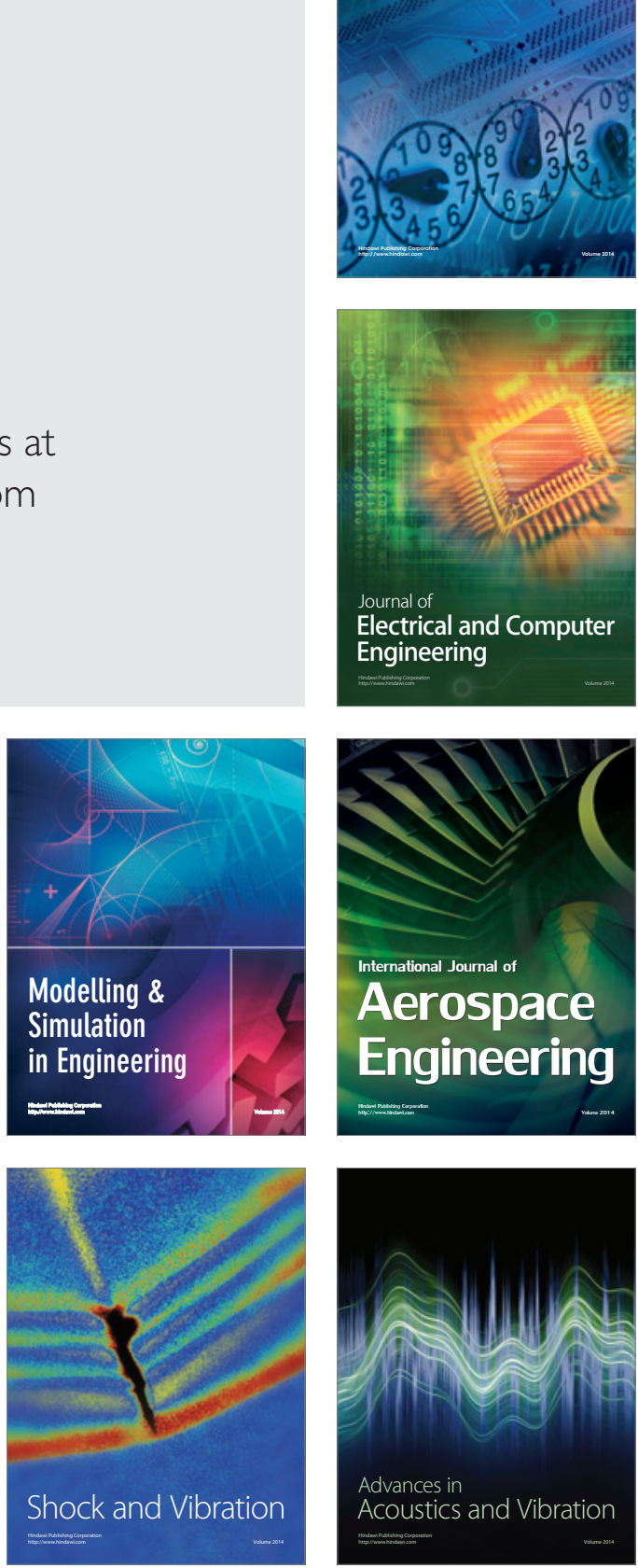